Article

\title{
LC-Q-TOF-MS/MS Identification of Specific Non-Meat Proteins and Peptides in Beef Burgers
}

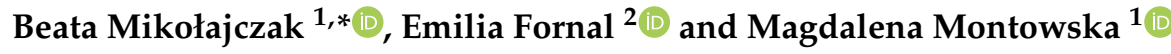 \\ 1 Department of Meat Technology, Poznan University of Life Sciences, Wojska Polskiego 31, \\ Poznań, 60-624, Poland; magdalena.montowska@up.poznan.pl \\ 2 Department of Pathophysiology, Medical University of Lublin, Jaczewskiego 8b, Lublin, 20-090, Poland; \\ emilia.fornal@umlub.pl \\ * Correspondence: beata.mikolajczak@up.poznan.pl; Tel.: +48-618-487-505
}

Academic Editors: Jacek Namieśnik and Justyna Płotka-Wasylka

Received: 3 December 2018; Accepted: 20 December 2018; Published: 21 December 2018

check for updates

\begin{abstract}
Beef burgers are a popular food choice, due to their taste and convenience. The extensive range of beef burgers with different flavours currently offered on the market is adding to their growing consumption. This study detected and identified specific non-meat proteins and peptide markers originating from functional preparations, i.e., powdered mixes of protein additives and spices, used as meat substitutes in the production of ready-to-cook beef burgers. Twenty-eight soy proteins, including isoforms (nine milk-, three pea- and one beetroot-specific protein) were found concurrently with a set of peptide markers unique to soy glycinin and $\beta$-conglycinin, pea vicilin and provicilin, milk $\alpha_{S 1}$-casein, $\beta$-lactoglobulin, as well as beetroot elongation factor 2 . Soy and beetroot proteins and peptides were observed in all burgers containing additives. Milk and pea proteins were included in powdered mixes but were not detected in burgers, indicating that their content was below the limit of detection. The study demonstrates that the proposed method can be implemented to analyse protein additives in cooked burgers; however, the presence of low amounts of additives, below $1-2 \%$, should be further confirmed by using a more sensitive triple quadrupole instrument.
\end{abstract}

Keywords: food authenticity; beef burgers; soy; pea; milk and beetroot proteins; allergenic proteins; peptide markers; LC-Q-TOF-MS/MS

\section{Introduction}

Food products of animal origin constitute one of the most important segments of the food economy worldwide. Global bovine production and its consumption are in third place, after poultry and pork, and have shown a continuing upward trend since 1970. Projected data show that beef consumption will increase by $1.8 \%$ in 2015-2030 [1]. Globalisation and the consequent increased exposure to Western culture, organisational changes, flexible working hours and the increasing presence of women in the workforce have influenced the shift in many consumer preferences and resulted in expanding consumer interest in convenience food. Beef burgers are among the most popular products in the convenience food market, owing to the taste and ease of preparation. The use of ready-to-cook or ready-to-eat burgers minimises the time and effort of the consumer required for meal preparation [2]. A survey conducted by Shan et al. [3] revealed that $20.2 \%$ of 481 consumers from the Republic of Ireland consumed beef burgers once a week while for $40.5 \%$ of the cohort, the frequency was 1-3 times per month.

Currently, there is a great demand for healthy meat products, that are, on the one hand, low in fat, cholesterol, salt, nitrites and energy content, but on the other hand, are enriched in health-promoting bioactive compounds, such as unsaturated fatty acids, sterols, bioactive peptides, flavonoids and fibre [4]. Therefore, manufacturers are exploring new functional preparations to develop high-quality 
products with low sodium content (below $1.2 \%$ ) as well as different sensory attributes, flavour and texture, according to consumer demands. Currently, burgers can be produced with interesting combinations of additives that besides reducing the costs, also enhance flavour and improve the quality characteristics. The addition of non-meat proteins (i.e., soy, milk, pea, egg white), aromatic herbs and spices, and extracts from plants and fruits, is becoming increasingly popular in beef burger production. These additives have different functional roles in the food matrix.

Legume proteins (soy, pea, bean) are commonly used in meat products to increase water holding capacity and improve the sensorial characteristics, such as texture (firmness, juiciness, sliceability). Soy proteins are also incorporated both as meat and animal fat substitutes for health reasons. These ingredients also serve an economic purpose-reducing the production cost while maintaining the high protein content in the product $[5,6]$. The addition of up to $3-5 \%$ soy protein increases the cooking yield and the weight of the final product [6,7].

All types of milk proteins, i.e., caseins, caseinates and whey proteins, and mixtures thereof, act as good water and fat binders and stabilisers. Various casein or whey ingredients are usually declared as "milk proteins" on the product label [8]. Previous work showed that milk proteins decreased the amount of free water and increased the immobilised water in comminuted frankfurter batters, thereby increasing the shear force value and firmness, and final textural modification of the frankfurters [9]. Among the milk proteins, $\beta$-casein is the most effective stabiliser because it reduces the surface tension to the greatest extent [10]. Moreover, enzymatic hydrolysates of soy or milk proteins contain various physiologically functional peptides [11]. For instance, the addition of $20 \mathrm{mg} / \mathrm{mL}$ casein peptides, obtained by the proteolytic enzymes Alcalase ${ }^{\circledR}$ and Flavourzyme ${ }^{\circledR}$, to ground beef homogenates, effectively inhibited lipid oxidation [12].

Various vegetable powders and extracts that are rich in natural antioxidants (i.e., flavonoids, phenolic compounds) enhance the healthful properties and quality of meat products [13]. For example, vegetable powders obtained from spinach, yellow pea, onion, red pepper, green pea and tomato improved the oxidative stability of cooked turkey meat patties by $20 \%$ to $30 \%$ [14]. Flavonoid extracts from beetroot proved to be effective in reducing lipid oxidation in cooked pork patties [15]. Beetroot extract is the main source of betalains-water-soluble pigments-primarily used as food colourants. Besides, betalains have strong antioxidative properties and may also exert other important activities, such as anticarcinogenic and antiglycation [16].

Substitution of ingredients is the most frequent food adulteration and, consequently, less valuable ingredients of non-meat origin can be illegally added to meat burgers, to replace the most expensive components. These ingredients may also be the source of undeclared allergens responsible for severe allergic or food intolerance responses in allergic or sensitive consumers. Since modern meat products consist of an increasing number of non-meat components, there is a need for novel, reliable and sensitive analytical methods, to ensure the accuracy of labelling, reveal dishonest producers and for consumer safety. Most commonly applied immunochemical- and DNA-based methods have some limitations, especially when analysing multi-component and thermally processed food matrices, while modern instrumental mass spectrometry (MS)-based methods, which are characterised by high resolving power, sensitivity and specificity, have succeeded in the analysis of complex and processed meat products [17].

In this paper, MS-based proteomic and peptidomic analyses are presented to detect and identify non-meat proteins and specific peptide markers originating from functional preparations used in the production of beef burgers. Ready-to-cook commercially produced burgers containing different meat content, non-meat proteins and other additives—namely, soy, pea and milk proteins-and powdered onion and beetroot juices were examined. Proteins and peptides specific to the observed additives were identified by liquid chromatography-quadrupole-time-of-flight-tandem mass spectrometry (LC-Q-TOF-MS/MS). 


\section{Results and Discussion}

\subsection{Identification of Specific Non-Meat Proteins}

Four types of industrially prepared, frozen, ready-to-cook beef burgers (samples B1-B4) were investigated in this study. The detailed composition of each product declared on the packaging label is shown in Table 1. It is well known that thermal treatment affects MS-based protein and peptide identification, due to protein denaturation, reduced protein solubility and aggregation processes. Therefore, in this work, the final identification and confirmation of the results were performed on cooked burgers (samples B5-B8), after storage at $-18{ }^{\circ} \mathrm{C}$ for $1 \mathrm{month}$, for the detection of unmodified, thermally-resistant proteins and peptides. The studied burgers were manufactured from beef incorporated with the addition of three different powdered mixes of functional additives and spices, which included, among others, soy, milk and pea proteins, powdered onion and beetroot juices, wheat fibre, plant flavours and spices, as shown in Table 1 . These three mixes were also analysed and used as reference samples M1-M3.

Table 1. The composition of beef burgers declared on the packaging.

\begin{tabular}{|c|c|c|c|c|}
\hline Ingredient (\%) & Burger B1/B5 & Burger B2/B6 & Burger B3/B7 & Burger B4/B8 \\
\hline Beef & 51 & 51 & 80 & 100 \\
\hline Fat & $\leq 20$ & $\leq 20$ & $\leq 20$ & $\leq 20$ \\
\hline Collagen & $\leq 25$ & $\leq 25$ & $\leq 25$ & $\leq 15$ \\
\hline Proteins & $\overline{11.5}$ & $\overline{13.1}$ & 16.0 & 19.0 \\
\hline $\mathrm{NaCl}$ & 0.95 & 1.1 & 0.8 & n.d. ${ }^{1}$ \\
\hline Mix & M1 & M2 & M3 & \\
\hline $\begin{array}{l}\text { Non-meat } \\
\text { proteins }\end{array}$ & $\begin{array}{c}\text { Soy proteins } \\
\text { rehydrated }(30 \%)\end{array}$ & $\begin{array}{l}\text { Soy proteins } \\
\text { rehydrated }(24 \%) \\
\text { Milk proteins }\end{array}$ & $\begin{array}{c}\text { Soy proteins } \\
\text { Pea proteins } \\
\text { Traces of milk proteins }\end{array}$ & n.d. \\
\hline $\begin{array}{l}\text { Other non-meat } \\
\text { additives }\end{array}$ & $\begin{array}{c}\text { Powdered onion } 1.78 \% \text {, } \\
\text { water, sodium chloride, } \\
\text { wheat fibre, plant } \\
\text { flavours, breadcrumbs, } \\
\text { monosodium } \\
\text { glutamate, powdered } \\
\text { beetroot juice, spices }\end{array}$ & $\begin{array}{l}\text { Water, sodium } \\
\text { chloride, wheat fibre, } \\
\text { plant flavours, } \\
\text { breadcrumbs, } \\
\text { powdered beetroot } \\
\text { juice, spices }\end{array}$ & $\begin{array}{c}\text { Water, sodium } \\
\text { chloride, plant } \\
\text { flavours, powdered } \\
\text { beetroot juice, dextrose }\end{array}$ & n.d. \\
\hline
\end{tabular}

Protein digests were analysed using LC-Q-TOF-MS/MS and the Spectrum Mill searching algorithm. More than 110 animal proteins, as well as 33-40 vegetable proteins, were identified, depending on the analysed samples. Burgers B4 (raw) and B8 (cooked) were made from 100\% meat. These burgers were used as the control groups for raw and cooked samples, respectively since they did not contain any functional preparations, as shown in Table 1 . In general, the same meat and vegetable proteins were revealed in raw and cooked burgers, but protein sequence coverage was lower in the cooked samples. Table 2 provides a comparison of the Spectrum Mill identification results for soy-specific proteins between powdered mixes of protein additives and spices (M1-M3) and the corresponding cooked burgers. There was a predominance of 20 soybean proteins, including glycinin (G1, G2, G3 and G4 isoforms) and $\beta$-conglycinin ( $\alpha, \alpha^{\prime}$ and $\beta$ chains) in all the examined samples, except for the control B4 and B8 burgers, characterised by a high $(>45.5 \%)$ sequence coverage, as shown in Table 2 . The same 20 soy proteins also occurred in raw burgers B1-B3. Soy proteins were partly substituted for meat in the burgers, which accounted for the large quantities of soy proteins present in the products. 
Table 2. Identification results for soy proteins from powdered mixes of protein additives (M1-M3) and corresponding cooked burgers (B5-B7).

\begin{tabular}{|c|c|c|c|c|c|c|c|c|c|c|c|c|c|c|}
\hline \multirow{2}{*}{ Identified Protein } & \multirow{2}{*}{$\begin{array}{c}\text { Accession } \\
\text { No. }\end{array}$} & \multirow{2}{*}{ Mass (Da) } & \multicolumn{3}{|c|}{ Matched Peptides $^{1}$} & \multicolumn{3}{|c|}{ Sequence Coverage $(\%)^{2}$} & \multicolumn{3}{|c|}{ Matched Peptides } & \multicolumn{3}{|c|}{ Sequence Coverage (\%) } \\
\hline & & & M1 & M2 & M3 & M1 & M2 & M3 & B5 & B6 & B7 & B5 & B6 & B7 \\
\hline Seed lipoxygenase-1 & P08170 & $94,595.8$ & 46 & 38 & 44 & 74.2 & 63.1 & 68.6 & 19 & 23 & 7 & 36.4 & 43.7 & 11.6 \\
\hline Seed lipoxygenase-2 & P09439 & $97,429.1$ & 47 & 34 & 45 & 73.8 & 52.7 & 71.5 & 18 & 5 & 7 & 28.5 & 5.9 & 9.3 \\
\hline Seed lipoxygenase-3 & P09186 & $97,155.8$ & 53 & 40 & 46 & 61.9 & 60 & 64 & 22 & 27 & 15 & 41.8 & 48.8 & 26.7 \\
\hline$\beta$-Conglycinin, $\alpha$ chain & P13916 & $70,577.1$ & 39 & 32 & 36 & 58.8 & 57 & 57 & 28 & 28 & 25 & 54.4 & 53.3 & 51.5 \\
\hline$\beta$-Conglycinin, $\alpha^{\prime}$ chain & P11827 & $74,609.2$ & 27 & 20 & 29 & 44.7 & 37.8 & 48.6 & 19 & 15 & 16 & 33.9 & 28.4 & 29.5 \\
\hline$\beta$-Conglycinin, $\beta$ chain & P25974 & $50,608.1$ & 29 & 27 & 28 & 68.1 & 66.9 & 68.1 & 18 & 21 & 14 & 59.9 & 61.2 & 48.9 \\
\hline Glycinin & P04347 & $54,960.3$ & 20 & 18 & 19 & 62 & 55.8 & 62 & 16 & 16 & 25 & 52.3 & 52.3 & 51.5 \\
\hline Glycinin G1 & P04776 & $54,868.3$ & 29 & 28 & 28 & 83 & 83 & 81.4 & 24 & 24 & 23 & 80.2 & 79.3 & 75.9 \\
\hline Glycinin G2 & P04405 & $64,042.4$ & 28 & 26 & 27 & 83.7 & 83.7 & 75.4 & 20 & 22 & 20 & 73.6 & 82.6 & 71.5 \\
\hline Glycinin G3 & P11828 & $58,411.5$ & 24 & 21 & 26 & 80 & 73.5 & 80 & 17 & 17 & 11 & 71.5 & 64.2 & 45.5 \\
\hline Glycinin G4 & P02858 & $56,332.9$ & 31 & 28 & 29 & 61.3 & 65.3 & 61.3 & 21 & 23 & 20 & 53.0 & 53.2 & 52.8 \\
\hline Sucrose-binding protein & Q04672 & $60,920.9$ & 24 & 18 & 26 & 56.1 & 45 & 58.9 & 16 & 18 & 13 & 41.4 & 47.5 & 29.3 \\
\hline Trypsin inhibitor A & P01070 & $24,290.1$ & 14 & 15 & 15 & 56.4 & 60.1 & 58.7 & 11 & 11 & 11 & 53.7 & 49 & 49.5 \\
\hline Kunitz-type trypsin inhibitor KTI1 & P25272 & $22,830.8$ & 9 & 8 & 11 & 47.7 & 36.9 & 54.1 & 5 & 4 & 4 & 34.4 & 23.6 & 23.6 \\
\hline$\beta$-Amylase & P10538 & $56,484.1$ & 14 & 10 & 14 & 39.1 & 30 & 35 & 3 & 3 & 1 & 10.2 & 10.2 & 5 \\
\hline Lectin & P05046 & $30,927.4$ & 10 & 11 & 10 & 71.9 & 72.9 & 71.9 & 8 & 8 & 5 & 60.7 & 64.2 & 35 \\
\hline $2 S$ albumin & P19594 & $19,030.2$ & 8 & 7 & 7 & 41.7 & 41.7 & 41.7 & 6 & 6 & 5 & 41.1 & 41.1 & 32.9 \\
\hline P24 oleosin isoform B & P29531 & $23,392.2$ & 7 & 7 & 7 & 30.4 & 30.4 & 30.4 & 4 & 3 & 3 & 18.8 & 13 & 13 \\
\hline P34 probable thiol protease & P22895 & $43,135.4$ & 6 & 6 & 6 & 22.1 & 22.1 & 22.1 & 5 & 5 & 4 & 21.8 & 21.8 & 21.6 \\
\hline Basic 7S globulin & P13917 & $47,133.9$ & 14 & 13 & 14 & 57.1 & 54.5 & 57.1 & 12 & 13 & 7 & 50.1 & 51.7 & 29.9 \\
\hline
\end{tabular}

${ }^{1}$ Number of matched peptides in the database search; ${ }^{2}$ Percentage of coverage of the protein amino acid sequence. 
Eight comparatively less abundant soy proteins, as well as pea-, milk- and beetroot-specific proteins found in powdered mixes of additives are presented in Table S1 (Supplementary Materials). Three pea-specific proteins, namely provicilin, vicilin and legumin, appeared only in mix M3. Nine milk proteins, i.e., $\beta$-lactoglobulin, $\alpha_{\mathrm{S} 1}$-casein, $\beta$-casein, lactotransferrin, butyrophilin, $\alpha$-lactalbumin, lactadherin, $\mathrm{k}$-casein and milk glycoprotein PP3, were observed in mix M2 but two whey proteins ( $\beta$-lactoglobulin and $\alpha$-lactalbumin) were detected in mix M3. These proteins were identified with good confidence scores, showing a sequence coverage in the range of 5.3-81.4 (milk proteins) and 3-13 (whey proteins) matched peptides. The results are consistent with the mixes' labels since the soy, milk or pea proteins were included in the list of ingredients for a given product. Possible traces of milk were declared on the M3 packaging. In addition, no onion proteins were found in the examined burgers and mixes (added to B1 and B5 at 1.78\%). Moreover, onion is rich in simple sugars while low in protein. The lack of pea, milk and onion protein content in the ready-to-cook burgers was most likely because these ingredients were present in quantities below the limit of detection of the method.

Powdered beetroot juice was declared in all three mixes of additives and, consequently, several beetroot proteins were found in all types of ready-to-cook beef burgers (B1-B3) manufactured with given mixes and their cooked counterparts (B5-B7). However, only one protein, namely, elongation factor 2 (EF-2), turned out to be unique to beetroot when its sequence was searched against all entries in the NCBI (National Centre for Biotechnology Information) protein sequence database for species specificity. Eukaryotic EF-2 is a protein with a calculated molecular weight of $94,767 \mathrm{Da}$ and 843 amino acid residues. It catalyses the guanosine triphosphatase-dependent ribosomal translocation step during translation elongation. In our mixes, the protein was identified with good confidence scores, 2-7 matched peptides and 3.9-10.9\% protein sequence coverage. Total ion chromatograms of the three mixes of additives and spices are shown in Figure S1 (Supplementary Materials), and total ion chromatograms of the cooked burgers manufactured with the addition of their respective mixes are shown in Figure S2 (Supplementary Materials).

Some of the major food allergens, such as glycinin (Gly m 6) and $\beta$-conglycinin (Gly $\mathrm{m} 5$ ) soy

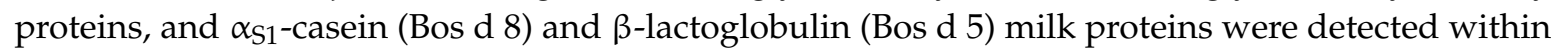
the study. These proteins have been determined previously in various commercial meat products, for example, frankfurters, chicken and pork liver sausage, and smoked and cooked chicken breast, by nano-LC-Q-TOF-MS/MS [17]. LC-QQQ-MS/MS in multiple reaction monitoring mode has been developed to detect soy (glycinin) and milk ( $\alpha_{S 1}$-casein, $\alpha_{S 2}$-casein) allergens in wheat bread spiked with soy flakes and skim milk powder [18]. Hoffmann et al. [19] noticed soy glycinin, and pea convicilin, vicilin and provicilin proteins in emulsion-type pork sausages, using a recently developed LC-MS/MS method. Allergies to pea proteins are less frequent than allergies to soy and mainly refer to the proteins vicilin and convicilin [20]. Vicilin and provicilin storage seed proteins were found in our mix M3 of functional additives and spices. Considering that neither pea nor milk proteins were detected in raw and cooked burgers, using our untargeted LC-Q-TOF-MS/MS method confirms that the results may be affected mostly by food complexity, the limit of detection of the chosen method and industrial processing [21]. It is recommended that a more sensitive and selective triple quadrupole mass spectrometer should further be implemented to analyse less abundant protein components in beef burgers. 


\subsection{Identification of Specific Peptide Markers}

The most intense, potentially unique, peptide markers of soy, cow milk, pea and beetroot occurring in the LC-Q-TOF-MS/MS profiles were searched for species and protein specificity, against all protein sequence records stored in the NCBI database, using the BLASTP algorithm. As a result, 47 unique peptides were most abundant, originating from soy glycinin G1, G2 and G3 isoforms and $\beta$-conglycinin, in all mixes, and raw and cooked beef burgers, as shown in Table 3. The Spectrum Mill protein score was over 449 , and the peptide intensity ranged from $10^{6}-10^{8}$. Figure S3 (Supplementary Materials) shows the mass spectrum of the peptide VFDGELQEGR $(m / z 575.28,2+)$ unique to soy glycinin $\mathrm{G} 1$ and obtained from a cooked burger (sample B5). For control groups of raw and cooked burgers (B4 and B8), no soy-, milk- and pea-specific peptides were discerned. These negative results confirm the correctness of the labelling.

Table 4 presents the peptides unique to cow milk, pea and beetroot obtained from powdered mixes of additives and spices. The peptides belong to some main food allergens, such as milk $\beta$-lactoglobulin, $\alpha$-lactalbumin and $\alpha_{\mathrm{S} 1}$-casein while two unique peptides derived from pea allergenic proteins vicilin and provicilin. For example, the MS/MS spectrum of the milk $\beta$-lactoglobulin marker peptide TPEVDDEALEK $(m / z 623.29,2+)$ obtained from mix M2 is presented in Figure 1. Considering the large number of specific peptides detected in this study, and the limited space available in this article, some of the peptides are presented in the Supplementary Materials. Peptides specific to both cow milk and milk of other mammals are shown in Table S2 (Supplementary Materials), and the soy-specific peptides obtained from less abundant proteins and detected in all mixes and burgers are shown in Table S3 (Supplementary Materials). Figure S4 (Supplementary Materials) presents the mass spectrum of the pea provicilin-specific peptide VLLEQQEQEPQHR $(m / z 545.28,3+)$ obtained from mix M3. 
Table 3. Peptides unique to glycinin G1, G2 and G3 isoforms, and $\beta$-conglycinin detected in all analysed samples of mixes and burgers.

\begin{tabular}{|c|c|c|c|c|c|c|c|}
\hline Parent Ion $(\mathrm{m} / \mathrm{z})$ & $\operatorname{Mr}(\exp )$ & $\operatorname{Exp} z^{1}$ & Peptide Score $^{2}$ & Total Intensity Range & Peptide Marker & Protein & Protein Score $^{3}$ \\
\hline 686.8540 & 1372.7002 & 2 & 19.99 & $1.23 \times 10^{6}-1.01 \times 10^{8}$ & (R)ALIQVVNCNGER(V) & \multirow{11}{*}{ Glycinin G1 } & \multirow{11}{*}{558.84} \\
\hline 765.3553 & 1529.7013 & 2 & 18.57 & $3.05 \times 10^{6}-7.95 \times 10^{7}$ & (R)EQPQQNECQIQK(L) & & \\
\hline 586.8197 & 1172.6310 & 2 & 20.15 & $1.87 \times 10^{7}-5.76 \times 10^{8}$ & (K)FLVPPQESQK $(R)$ & & \\
\hline 889.7510 & 2667.2330 & 3 & 22.57 & $1.84 \times 10^{7}-8.57 \times 10^{8}$ & (K)GIFGMIYPGCPSTFEEPQQPQQR(G) & & \\
\hline 820.1402 & 3277.5284 & 4 & 22.42 & $3.19 \times 10^{7}-9.40 \times 10^{8}$ & (K)HQQEEENEGGSILSGFTLEFLEHAFSVDK(Q) & & \\
\hline 554.3123 & 1107.6157 & 2 & 19.15 & $2.14 \times 10^{5}-1.10 \times 10^{7}$ & (R)LSAEFGSLR(N) & & \\
\hline 616.7735 & 1232.5390 & 2 & 16.95 & $1.22 \times 10^{6}-3.56 \times 10^{7}$ & (K)NLQGENEGEDK(G) & & \\
\hline 1141.5955 & 3422.7624 & 3 & 22.17 & $1.76 \times 10^{7}-1.60 \times 10^{9}$ & (K)TNDTPMIGTLAGANSLLNALPEEVIQHTFNLK(S) & & \\
\hline 575.2810 & 1149.5535 & 2 & 21.65 & $3.21 \times 10^{6}-2.30 \times 10^{8}$ & (R)VFDGELQEGR(V) & & \\
\hline 713.4353 & 1425.8576 & 2 & 24.28 & $2.64 \times 10^{7}-4.45 \times 10^{8}$ & (R)VLIVPQNFVVAAR(S) & & \\
\hline 709.3268 & 1417.6455 & 2 & 19.51 & $1.15 \times 10^{5}-1.90 \times 10^{7}$ & (K)YQQEQGGHQSQK(G) & & \\
\hline 793.8984 & 1586.7849 & 2 & 23.48 & $1.53 \times 10^{7}-3.68 \times 10^{8}$ & (R)FYLAGNQEQEFLK(Y) & \multirow{4}{*}{$\begin{array}{c}\text { Glycinin G1/G2 } \\
\text { G1-G3 } \\
\text { Glycinin G1/G3 }\end{array}$} & $558.84 / 549.21$ \\
\hline 725.8287 & 1450.6485 & 2 & 21.39 & $1.94 \times 10^{7}-3.82 \times 10^{8}$ & (R)SQSDNFEYVSFK(T) & & $558 / 549 / 449$ \\
\hline 1246.6297 & 2492.2503 & 2 & 22.62 & $9.73 \times 10^{5}-1.61 \times 10^{8}$ & (K)NAMFVPHYNLNANSIIYALNGR(A) & & \\
\hline 679.8461 & 1358.6845 & 2 & 19.95 & $6.93 \times 10^{5}-6.69 \times 10^{7}$ & (R)ALVQVVNCNGER(V) & & $558.84 / 449.67$ \\
\hline 632.3305 & 1263.6514 & 2 & 19.95 & $1.33 \times 10^{6}-3.23 \times 10^{8}$ & (K)EAFGVNMQIVR(N) & \multirow{8}{*}{ Glycinin G2 } & \multirow{7}{*}{549.21} \\
\hline 897.7005 & 2691.0846 & 3 & 19.95 & $4.33 \times 10^{5}-1.16 \times 10^{8}$ & (R)KPQQEEDDDDEEEQPQCVETDK(G) & & \\
\hline 497.7697 & 994.5316 & 2 & 18.63 & $4.55 \times 10^{6}-8.60 \times 10^{7}$ & (K)LSAQYGSLR(K) & & \\
\hline 1240.1321 & 2479.2551 & 2 & 20.70 & $1.54 \times 10^{6}-1.56 \times 10^{8}$ & (K)NAMFVPHYTLNANSIIYALNGR(A) & & \\
\hline 966.4660 & 1931.9193 & 2 & 24.50 & $1.12 \times 10^{7}-6.50 \times 10^{8}$ & (R)NLQGENEEEDSGAIVTVK $(\mathrm{G})$ & & \\
\hline 937.4649 & 1873.9191 & 2 & 20.23 & $1.11 \times 10^{7}-6.50 \times 10^{8}$ & (K)NNNPFSFLVPPQESQR(R) & & \\
\hline 915.9747 & 3660.8544 & 3 & 19.69 & $1.03 \times 10^{7}-4.26 \times 10^{8}$ & (R)QNIGQNSSPDIYNPQAGSITTATSLDFPALWLLK(L) & & \\
\hline 1183.5625 & 2366.1147 & 2 & 22.92 & $1.41 \times 10^{7}-5.19 \times 10^{8}$ & (K)QQEEENEGSNILSGFAPEFLK(E) & & \\
\hline 1201.1432 & 2401.2762 & 2 & 22.88 & $3.55 \times 10^{7}-9.17 \times 10^{8}$ & (R)VFDGELQEGGVLIVPQNFAVAAK(S) & \multirow[t]{2}{*}{ Glycinin G2 } & \\
\hline 747.8511 & 1494.6932 & 2 & 17.89 & $3.91 \times 10^{5}-3.49 \times 10^{7}$ & (K)YQQQQQGGSQSQK $(\mathrm{G})$ & & 549.21 \\
\hline 1116.0425 & 2231.0768 & 2 & 20.69 & $4.61 \times 10^{5}-7.80 \times 10^{7}$ & (R)FYLAGNQEQEFLQYQPQK(Q) & \multirow[t]{4}{*}{ Glycinin G3 } & \\
\hline 1205.6108 & 3614.8126 & 3 & 20.39 & $3.05 \times 10^{6}-2.46 \times 10^{8}$ & (R)HNIGQTSSPDIFNPQAGSITTATSLDFPALSWLK(L) & & \\
\hline 1045.4962 & 3134.4701 & 3 & 24.92 & $8.48 \times 10^{5}-1.43 \times 10^{8}$ & (R)QQEEENEGGSILSGFAPEFLEHAFVVDR $(\mathrm{Q})$ & & 449.67 \\
\hline 867.2119 & 3465.8085 & 4 & 19.47 & $1.11 \times 10^{6}-2.79 \times 10^{7}$ & (K)TNDRPSIGNLAGANSLLNALPEEVIQQTFNLR(R) & & \\
\hline 1047.1164 & 2093.2216 & 2 & 22.67 & $3.90 \times 10^{7}-2.59 \times 10^{8}$ & (K)AIVILVINEGDANIELVGLK $(\mathrm{K})$ & \multirow{7}{*}{$\begin{array}{c}\beta \text {-Conglycinin, } \\
\alpha \text { chain }\end{array}$} & \multirow{7}{*}{675.52} \\
\hline 901.1371 & 2701.3906 & 3 & 22.17 & $1.31 \times 10^{7}-3.61 \times 10^{8}$ & (R)DLDIFLSIVDMNEGALLLPHFNSK(A) & & \\
\hline 1013.4783 & 2025.9472 & 2 & 23.37 & $3.79 \times 10^{6}-1.48 \times 10^{8}$ & (K)EQQQEQQQEEQPLEVR(K) & & \\
\hline 592.2857 & 1183.5630 & 2 & 16.74 & $2.38 \times 10^{6}-1.38 \times 10^{8}$ & (R)ESYFVDAQPK $(\mathrm{K})$ & & \\
\hline 1124.9099 & 3372.7070 & 3 & 19.96 & $2.48 \times 10^{7}-1.45 \times 10^{9}$ & (R)NFLAGSQDNVISQIPSQVQELAFPGSAQAVEK(L) & & \\
\hline 526.2697 & 1051.5320 & 2 & 14.05 & $2.03 \times 10^{6}-2.36 \times 10^{8}$ & (K)NPFLFGSNR(F) & & \\
\hline 1076.5199 & 2152.0305 & 2 & 23.11 & $2.01 \times 10^{7}-5.28 \times 10^{8}$ & (R)VPSGTTYYVVNPDNNENLR(L) & & \\
\hline
\end{tabular}


Table 3. Cont

\begin{tabular}{|c|c|c|c|c|c|c|c|}
\hline Parent Ion $(\mathrm{m} / \mathrm{z})$ & Mr (exp) & $\operatorname{Exp} z^{1}$ & Peptide Score $^{2}$ & Total Intensity Range & Peptide Marker & Protein & Protein Score $^{3}$ \\
\hline 622.8594 & 1244.7096 & 2 & 20.04 & $1.31 \times 10^{7}-2.47 \times 10^{8}$ & (R)LQESVIVEISK(E) & $\beta$-Conglycinin, & \\
\hline 577.2909 & 1153.5735 & 2 & 18.62 & $5.51 \times 10^{6}-1.82 \times 10^{8}$ & (R)NILEASYDTK(F) & $\alpha / \alpha^{\prime}$ chain & $675.52 / 482.98$ \\
\hline 698.4148 & 2093.2216 & 3 & 18.22 & $7.53 \times 10^{6}-4.52 \times 10^{7}$ & (K)AIVVLVINEGEANIELVGIK(E) & & \\
\hline 903.1223 & 2707.3436 & 3 & 21.01 & $5.11 \times 10^{6}-3.15 \times 10^{8}$ & (R)DLDVFLSVVDMNEGALFLPHFNSK(A) & $\beta$-Conglycinin, & \\
\hline 669.8188 & 1338.6284 & 2 & 18.65 & $9.42 \times 10^{5}-1.41 \times 10^{7}$ & (R)DSYNLQSGDALR(V) & $\alpha^{\prime}$ chain & 482.98 \\
\hline 1215.5866 & 2430.1612 & 2 & 21.58 & $4.20 \times 10^{5}-1.29 \times 10^{8}$ & (R)FESFFLSSTQAQQSYLQGFSK(N) & & \\
\hline 1047.1164 & 2093.2216 & 2 & 22.67 & $3.90 \times 10^{7}-2.59 \times 10^{8}$ & (K)AIVILVINEGDANIELVGIK(E) & $\beta$-Conglycinin, & \\
\hline 886.4683 & 2657.3821 & 3 & 22.61 & $6.34 \times 10^{6}-2.00 \times 10^{8}$ & (R)DLDIFLSSVDINEGALLLPHFNSK(A) & $\beta$ chain & \\
\hline 615.3408 & 1229.6736 & 2 & 16.74 & $1.54 \times 10^{6}-1.16 \times 10^{8}$ & (R)QQEGVIVELSK(E) & & 546.79 \\
\hline 887.4435 & 1773.8766 & 2 & 18.78 & $1.79 \times 10^{6}-1.12 \times 10^{8}$ & (R)QVQELAFPGSAQDVER(L) & & \\
\hline 478.7618 & 956.5160 & 2 & 17.37 & $1.40 \times 10^{6}-5.29 \times 10^{7}$ & (R)SPQLENLR(D) & & \\
\hline
\end{tabular}

Table 4. Peptides specific to cow milk, pea and beetroot obtained from mixes of additives.

\begin{tabular}{|c|c|c|c|c|c|c|c|}
\hline Parent Ion $(\mathrm{m} / \mathrm{z})$ & Mr (exp) & $\operatorname{Exp} z$ & Peptide Score & Total Intensity Range & Peptide Marker & Protein & Protein Score \\
\hline \multicolumn{8}{|c|}{ Milk Peptides } \\
\hline 858.4109 & 1715.8057 & 2 & 20.69 & $8.80 \times 10^{6}-6.91 \times 10^{8}$ & (R)LSFNPTQLEEQCHI(-) & \multirow{2}{*}{$\beta$-Lactoglobulin } & \multirow{2}{*}{245.95} \\
\hline 623.2994 & 1245.5845 & 2 & 22.62 & $4.95 \times 10^{7}-1.49 \times 10^{8}$ & (R)TPEVDDEALEK(F) & & \\
\hline 772.7189 & 2316.1369 & 3 & 18.97 & $3.77 \times 10^{7}-4.27 \times 10^{7}$ & (K)EPMIGVNQELAYFYPELFR(Q) & \multirow{3}{*}{$\alpha_{\mathrm{S} 1}$-Casein } & \multirow{3}{*}{106.74} \\
\hline 692.8696 & 1384.7300 & 2 & 18.97 & $4.15 \times 10^{7}-4.61 \times 10^{7}$ & (R)FFVAPFPEVFGK(E) & & \\
\hline 880.4773 & 1759.9450 & 2 & 20.53 & $4.35 \times 10^{7}-4.82 \times 10^{7}$ & (K)HQGLPQEVLNENLLR(F) & & \\
\hline 374.2062 & 747.4036 & 2 & 10.70 & $3.30 \times 10^{5}-7.24 \times 10^{5}$ & (R)APVDAFK(E) & Lactotransferrin & 56.56 \\
\hline 638.8128 & 1276.6168 & 2 & 17.53 & $1.09 \times 10^{6}-1.19 \times 10^{6}$ & (R)NPDEEGLFTVR(A) & \multirow{4}{*}{$\begin{array}{l}\text { Butyrophilin subfamily } 1 \\
\text { member A1 } \\
\text { Lactadherin }\end{array}$} & \multirow{2}{*}{80.89} \\
\hline 481.2771 & 961.5465 & 2 & 16.66 & $1.11 \times 10^{6}-2.21 \times 10^{6}$ & (K)VSPAVFVSR(E) & & \\
\hline 854.9494 & 1708.8905 & 2 & 14.72 & $9.73 \times 10^{5}-2.10 \times 10^{6}$ & (K)INLFDTPLETQYVR(L) & & 40.87 \\
\hline 374.2060 & 1120.6010 & 3 & 14.82 & $4.33 \times 10^{5}-7.64 \times 10^{5}$ & (R)IQPVAWHNR(I) & & \multirow{4}{*}{$\begin{array}{l}34.99 \\
43.40\end{array}$} \\
\hline 990.5502 & 1980.0913 & 2 & 16.02 & $6.20 \times 10^{6}-1.21 \times 10^{7}$ & (R)SPAQILQWQVLSNTVPAK(S) & \multirow{3}{*}{$\begin{array}{c}\text { k-Casein } \\
\text { Glycosylation-dependent cell } \\
\text { adhesion molecule } 1\end{array}$} & \\
\hline 630.8260 & 1260.6430 & 2 & 14.40 & $3.86 \times 10^{6}-4.31 \times 10^{6}$ & (R)NLQISNEDLSK(E) & & \\
\hline 421.8969 & 1263.6732 & 3 & 16.35 & $1.23 \times 10^{7}-1.32 \times 10^{7}$ & (K)SLFSHAFEVVK(T) & & \\
\hline \multicolumn{8}{|c|}{ Pea Peptides } \\
\hline 545.2826 & 1633.8293 & 3 & 12.27 & $5.19 \times 10^{5}-9.63 \times 10^{5}$ & (K)VLLEQQEQEPQHR(R) & Provicilin (Fragment) & 59.72 \\
\hline 957.9525 & 1914.8967 & 2 & 12.61 & $1.06 \times 10^{6}-1.16 \times 10^{6}$ & (K)NILEASFNTDYEEIEK(V) & Vicilin & 47.03 \\
\hline \multicolumn{8}{|c|}{ Beetroot Peptides } \\
\hline 753.0750 & 2257.2034 & 3 & 21.82 & $1.09 \times 10^{6}-9.85 \times 10^{6}$ & (K)STLTDSLVAAAGIIAQEVAGDVR(M) & \multirow{4}{*}{$\begin{array}{c}\text { Elongation factor } 2 \\
\text { (EF-2) }\end{array}$} & \multirow{4}{*}{88.19} \\
\hline 974.1526 & 2920.4649 & 3 & 15.64 & $2.03 \times 10^{6}-2.11 \times 10^{6}$ & (K)VIENANVIMATYEDPLLGDVQVYPEK(G) & & \\
\hline 560.2594 & 1119.5099 & 2 & 14.72 & $2.90 \times 10^{5}-7.04 \times 10^{5}$ & (K)EGALAEENMR(G) & & \\
\hline 372.6955 & 744.3828 & 2 & 11.03 & $8.55 \times 10^{5}-1.69 \times 10^{6}$ & (R)FFAFGR(V) & & \\
\hline
\end{tabular}




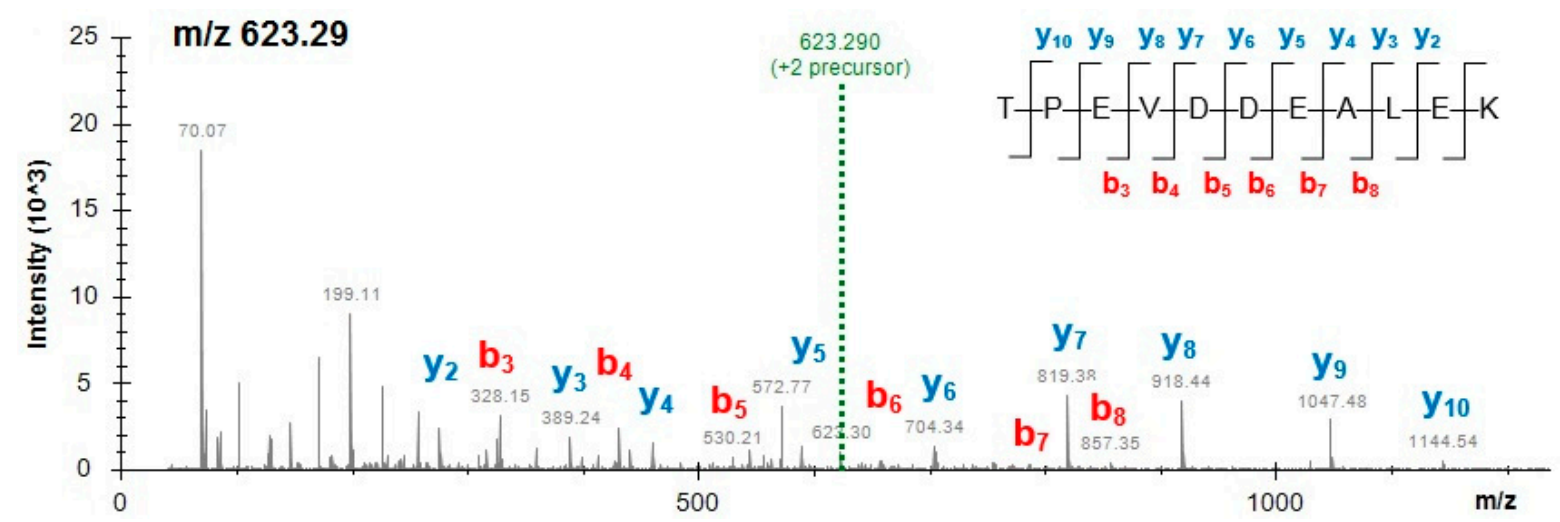

Figure 1. Mass spectrum of the milk $\beta$-lactoglobulin marker peptide TPEVDDEALEK (2+) obtained from functional additive preparation (mix M2).

Among four unique peptides originating from beetroot EF-2 protein, as shown in Table 4, only one peptide STLTDSLVAAAGIIAQEVAGDVR $(\mathrm{m} / \mathrm{z} 753.07,3+)$ was detected in all samples of mixes and burgers, as shown in Figure 2. The other three peptides were identified in powdered mixes. Figures S5 and S6 (Supplementary Materials) display the mass spectra of the peptides unique to beetroot EF-2, that is, EGALAEENMR $(m / z 560.25,2+)$ and FFAFGR $(m / z 372.69,2+)$, respectively, acquired from mix M1. Powdered beetroot juice was added to the examined burgers containing a reduced meat content, to enhance the redness in products that incorporated a significant amount of meat replacers (i.e., soy, pea and milk protein additives, Table 1). Beetroot extracts are an increasingly popular component in the meat industry not only for their colour but also because of their antioxidant activity and positive impact on the preservation of the final meat product $[14,15,22]$.

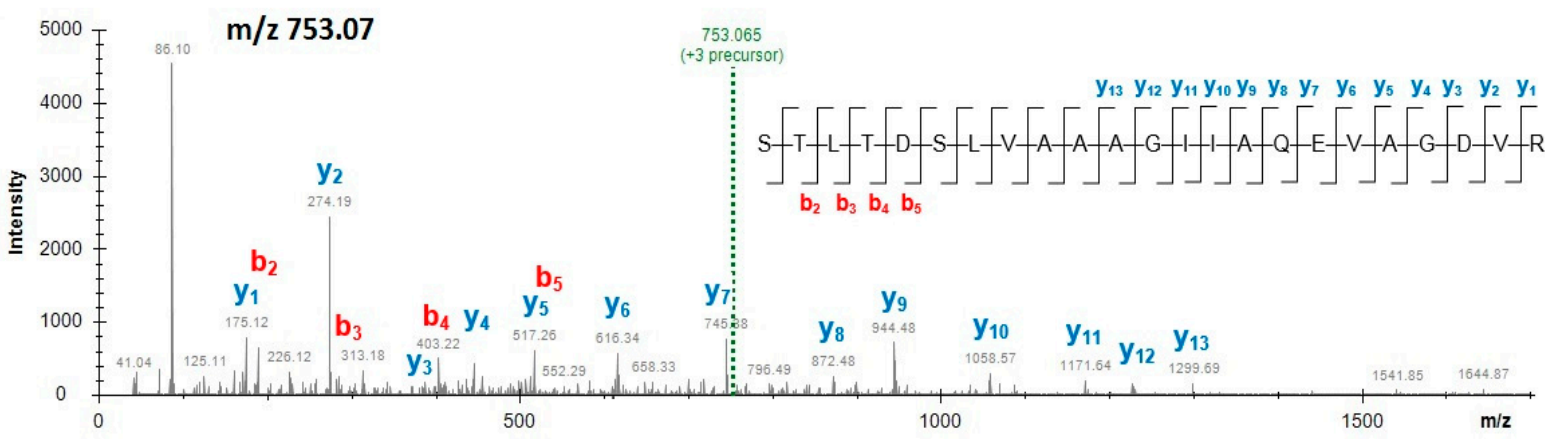

Figure 2. Mass spectrum of the beetroot elongation factor 2-specific peptide STLTDSLVAAAGIIAQEVAGDVR (3+) obtained from functional additive preparation (mix M1).

Unique peptides derived from non-meat proteins may be implemented for the simultaneous detection of soy, pea, milk and beetroot in complex food matrices. Previously, the same two soy peptides FYLAGNQEQEFLK (glycinin G1/G2) and SQSDNFEYVSFK (glycinin G1/G2/G3) found in this work, have been used to detect soy alongside six other allergenic commodities (milk, egg, hazelnut, peanut, walnut and almond) in bread [18], as well as soy, alongside egg, milk, hazelnut and peanut, in spiked cookie samples [23]. Peptide markers FYLAGNQEQEFLK (glycinin G1/G2), EAFGVNMQIVR (glycinin G2) and HFLAQSFNTNEDIAEK (glycinin G4) have been identified in various types of poultry meat products manufactured with the addition of functional protein preparations [17]. The same three soy peptides have been chosen for soy detection in emulsion-type sausages spiked with soy protein isolate [19]. Recently, the simultaneous quantification of meat and allergenic protein additives, including soy and milk, was conducted in a wide range of meat products (raw, cooked, smoked, sterilised), based on two glycinin marker peptides VLIVPQNFVVAAR (G1) and ISTLNSLTLPALR (G4), as well as bovine $\alpha_{S 1}$-casein peptides YLGYLEQLLR and FFVAPFPEVFGK [24]. Moreover, some of the whey peptides presented herein, have been identified in meat products, cookies 
and dairy products $[17,23,25,26]$. The results of the present study confirm the applicability of MS-based methods to detect multiple components and allergens in beef burgers.

Mass spectrometry is a robust technique that can differentiate between accidental contamination that may be a source of hidden allergens and intentional addition to meat products prepared with non-meat ingredients used for the replacement of the most expensive components of beef burgers, and thus is a reliable technique to detect meat adulterations [17-19,24]. However, to increase the sensitivity of the analysis, the method should be transferred to a more sensitive QQQ mass spectrometer and validated in multiple reaction monitoring mode.

\section{Materials and Methods}

\subsection{Preparation of Samples}

Four types of commercially-available, ready-to-cook, frozen beef burgers were manufactured in a local meat processing plant in the Wielkopolska region, Poland. Each packet contained 10 eighty-gram burgers of $1 \mathrm{~cm}$ thickness. After purchase, the frozen burgers were coded, vacuum-packed in polyethylene bags and kept at $-18{ }^{\circ} \mathrm{C}$ for future peptidomic analyses. Three burgers from each type of beef burgers were analysed. Each type differed mainly in the content of meat, protein additives and spices. Table 1 lists the detailed composition of the examined burgers presented on the product label. Minced beef used for the manufacture of burgers met the requirements of the European Union Regulation 1169/2011 [8] concerning the content of fat $(\leq 20 \%)$ and the collagen/meat proteins ratio $(\leq 15 \%)$. The beef content in the control group B4 was $100 \%$. Proteins of five species, namely, soy (Glycine max), cow milk (Bos taurus), pea (Pisum sativum), onion (Allium cepa) and beetroot (Beta vulgaris) were examined in the present study.

All types of burger samples were heated in a convection-steam oven (model SCC 61E, Rational International AG, Heerbrugg, Germany) at $180^{\circ} \mathrm{C}$ for 9 min. Raw (samples B1-B4) and cooked beef burgers (B5-B8) were analysed in triplicate. Additionally, three powdered mixes of functional preparations, consisting of various protein additives and spices (samples M1-M3) that were used to produce the beef burgers, as shown in Table 1, were analysed as reference samples.

\subsection{Protein Digestion}

Samples $(0.3 \mathrm{~g})$ were homogenised in $0.1 \mathrm{~mol} / \mathrm{L}$ of aqueous ammonium bicarbonate, using a T25 Ultra-Turrax (IKA Labortechnik, Staufen, Germany) at $9500 \mathrm{rpm}$ for $2 \times 20 \mathrm{~s}$ and then vacuum-dried using a miVac Duo Concentrator (Genevac Ltd., Ipswich, UK). Dried samples of functional preparations $(5 \mathrm{mg})$ and burgers $(10 \mathrm{mg})$ were rehydrated in $100 \mu \mathrm{L}$ of $0.1 \mathrm{~mol} / \mathrm{L}$ ammonium bicarbonate $(1 \mathrm{~h})$. The proteins were reduced by $0.2 \mathrm{~mol} / \mathrm{L}$ dithiothreitol (DTT) at $56^{\circ} \mathrm{C}$ for $1 \mathrm{~h}$ and then alkylated using iodoacetamide (IAA), at room temperature for $30 \mathrm{~min}$ in the dark. The remaining IAA was quenched by the addition of $0.2 \mathrm{~mol} / \mathrm{L}$ DTT and incubation at room temperature for $30 \mathrm{~min}$. The samples were digested in an ammonium bicarbonate solution ( $\mathrm{pH} 8.3$ ) containing $0.083 \mu \mathrm{g} / \mu \mathrm{L}$ trypsin, at $37^{\circ} \mathrm{C}$, overnight $(18 \mathrm{~h})$. The digests were purified by reversed-phase extraction, using Sep-Pak C18 Plus cartridges (Waters, Milford, MA, USA). The dried (miVac Duo concentrator) eluates were resuspended in $0.1 \%$ formic acid in milli-Q water (solvent $\mathrm{A}$ ), before analysis by LC-Q-TOF-MS/MS.

\subsection{LC-Q-TOF-MS/MS Analysis}

The ultra-high-performance LC-Q-TOF-MS/MS analysis was carried out using an Agilent Technologies (Santa Clara, CA, USA) 1290 Infinity Series liquid chromatograph coupled with an Agilent Technologies 6550 iFunnel Q-TOF LC/MS device equipped with an electrospray ionisation Agilent Technologies Jet Stream ion source. Chromatographic separation was achieved on an Agilent Zorbax Eclipse Plus C18 RRHD column $(2.1 \times 150 \mathrm{~mm}, 1.8 \mu \mathrm{m}$ pore size) (Agilent, Santa Clara, CA, USA). The injection volume was $10 \mu \mathrm{L}$. The mobile phase consisted of $0.1 \%$ formic acid in milli-Q water (solvent A) and $0.1 \%$ formic acid in $98 \%$ acetonitrile/milli-Q water (solvent B) at a flow rate of 
$0.3 \mathrm{~mL} / \mathrm{min}$. The mobile phase gradient (3-97\% B) steps were applied as follows: $0-1 \mathrm{~min}, 3 \% \mathrm{~B}$; 1-40 $\mathrm{min}, 5 \% \mathrm{~B} ; 40-45 \mathrm{~min}, 40 \% \mathrm{~B} ; 45-55 \mathrm{~min}, 90 \% \mathrm{~B}$, and a $5 \mathrm{~min}$ post-run at 3\% B. The source nitrogen gas temperature was $250{ }^{\circ} \mathrm{C}$, the sheath gas flow was $11 \mathrm{~L} / \mathrm{min}$, and the nebuliser pressure was 35 psig. The voltages were set at 3500 (capillary), 1000 (nozzle) and $400 \mathrm{~V}$ (fragmentor). Positive ions were acquired in the range of 100-1700 m/z for MS scans, and 40-1700 m/z for auto MS/MS scans, at a scan rate of 5 scans/s for MS and 3 scans/s for MS/MS, respectively. Internal mass correction was enabled, by using two reference masses at 121.0509 and $922.0098 \mathrm{~m} / \mathrm{z}$. Instrument control and data acquisition were performed using Agilent MassHunter Workstation software.

\subsection{Protein and Peptide Identification}

A UniProtKB/Swiss-Prot database search for protein and peptide identification was performed, using the Spectrum Mill MS Proteomics Workbench, using a 20 ppm precursor mass tolerance, 50 ppm product mass tolerance and $>70 \%$ precursor isolation purity. Search parameters were set as follows: trypsin enzyme, taxonomy all entries, one missed cleavage, carbamidomethylation as fixed modification, methionine oxidation as a variable modification and $>70 \%$ score peak intensity. The matches and Spectrum Mill scores were evaluated at $1 \%$ of the false discovery rate (FDR), for identity and homology threshold. Protein identification corresponding to protein sequences with a minimum of two unique peptides was accepted $[27,28]$. Selected peptides in FASTA format (amino acid residues are represented using single-letter codes) were searched against the NCBI non-redundant protein database using the protein BLAST alignment research tool and BLASTP algorithm (USA National Library of Medicine, Bethesda, MD, USA), for species and protein specificity [29].

\section{Conclusions}

Beef burgers - convenience food products with a high frequency of consumption-should be of good quality, which relies on close monitoring by adequate food agencies and regulatory bodies. The escalating inclusion of non-meat ingredients in meat products, on the one hand, contributes to increasing the range of products on the market and the market size, and on the other hand, can add to the growing number of cases of food intolerance. In this study, an LC-Q-TOF-MS/MS method for multi-component detection of soy, pea, milk and beetroot in ready-to-cook and cooked beef burgers supplemented with functional preparations was examined. Specific soy, pea, milk and beetroot proteins were detected, including a set of unique peptides originating from allergenic proteins, namely, soy glycinin and $\beta$-conglycinin, pea vicilin and provicilin, and milk $\alpha_{\mathrm{S1}}$-casein and $\beta$-lactoglobulin. These peptides can be used as peptide markers of food authenticity. The method can be implemented to study complex and processed food matrices, and thus can be an alternative approach to various enzyme-linked immunosorbent assays and polymerase chain reaction tests.

Supplementary Materials: The following are available online. Table S1. Soy, pea, milk and beetroot specific proteins detected in powdered mixes of additives; Table S2. Peptides specific to cow milk and milk of other mammals; Table S3. Soy unique peptides obtained from less abundant proteins and detected in all mixes and burgers; Figure S1. Total ion chromatograms of the mixes of functional preparations obtained from in-solution tryptic digests: (A) mix M1 with powdered onion as main differentiating component; (B) mix M2 with milk proteins as main differentiating component; (C) mix M3 with pea proteins as main differentiating component. All three mixes contained also soy proteins and powdered beetroot juice; Figure S2. Total ion chromatograms of the cooked burgers manufactured with the addition of mixes of functional preparations: (A) burger B1 containing mix M1 with powdered onion as main differentiating component; (B) burger B2 containing mix M2 with milk proteins as main differentiating component; $(\mathrm{C})$ burger B3 containing mix M3 with pea proteins as main differentiating component; Figure S3. Mass spectrum of the soy glycinin G1 unique peptide VFDGELQEGR (2+) obtained from cooked burger (sample B5); Figure S4. Mass spectrum of the pea provicilin unique peptide VLLEQQEQEPQHR $(3+)$ obtained from functional additive preparation ( $\operatorname{mix} \mathrm{M} 3$ ); Figure S5. Mass spectrum of the beetroot elongation factor 2 unique peptide EGALAEENMR (2+) obtained from functional additive preparation (mix M1); Figure S6. Mass spectrum of the beetroot elongation factor 2 unique peptide FFAFGR (2+) obtained from functional additive preparation (mix M1). 
Author Contributions: B.M. and M.M. designed the research; B.M. and E.F. performed the experiments and data analysis; M.M. helped in the data analysis and prepared figures; B.M. and M.M. interpreted data and wrote the paper. All authors discussed, edited and approved the final version.

Funding: This research received no external funding.

Conflicts of Interest: The authors declare no conflict of interest.

\section{References}

1. Alexandratos, N.; Jorge, M. World Agriculture: Towards 2015/2030 An FAO Perspective. Available online: http:/ / www.fao.org/docrep/005/y4252e/y4252e05b.htm (accessed on 17 December 2018).

2. Brunner, T.A.; van der Horst, K.; Siegrist, M. Convenience food products. Drivers for consumption. Appetite 2010, 55, 498-506. [CrossRef]

3. Shan, L.C.; De Brún, A.; Henchion, M.; Li, C.; Murrin, C.; Wall, P.G.; Monahan, F.J. Consumer evaluations of processed meat products reformulated to be healthier-A conjoint analysis study. Meat Sci. 2017, 131, 82-89. [CrossRef] [PubMed]

4. Viana, M.M.; dos Santos Silva, V.L.; Trindade, M.A. Consumers' perception of beef burgers with different healthy attributes. LWT Food Sci. Technol. 2014, 59, 1227-1232. [CrossRef]

5. Castro-Rubio, F.; García, M.C.; Rodríguez, R.; Marina, M.L. Simple and Inexpensive Method for the Reliable Determination of Additions of Soybean Proteins in Heat-Processed Meat Products: An Alternative to the AOAC Official Method. J. Agric. Food Chem. 2005, 53, 220-226. [CrossRef] [PubMed]

6. Carvalho, G.R.d.; Milani, T.M.G.; Trinca, N.R.R.; Nagai, L.Y.; Barretto, A.C.d.S. Textured soy protein, collagen and maltodextrin as extenders to improve the physicochemical and sensory properties of beef burger. Food Sci. Technol. 2017, 37, 10-16. [CrossRef]

7. Cassini, A.S.; Marczak, L.D.F.; Noreña, C.P.Z. Drying Characteristics of Textured Soy Protein: A Comparison between Three Different Products. Dry. Technol. 2007, 25, 2047-2054. [CrossRef]

8. Official Journal of the European Union Regulation UE No 1169/2011. Off. J. Eur. Union 2011, 54.

9. Hung, S.C.; Zayas, J.F. Functionality of Milk Proteins and Corn Germ Protein Flour in Comminuted Meat Products. J. Food Qual. 1992, 15, 139-152. [CrossRef]

10. Darewicz, M.; Dziuba, B.; Minkiewicz, P.; Dziuba, J. The Preventive Potential of Milk and Colostrum Proteins and Protein Fragments. Food Rev. Int. 2011, 27, 357-388. [CrossRef]

11. Arihara, K. Strategies for designing novel functional meat products. Meat Sci. 2006, 74, 219-229. [CrossRef]

12. Rossini, K.; Noreña, C.P.Z.; Cladera-Olivera, F.; Brandelli, A. Casein peptides with inhibitory activity on lipid oxidation in beef homogenates and mechanically deboned poultry meat. LWT Food Sci. Technol. 2009, 42, 862-867. [CrossRef]

13. Kumar, Y.; Yadav, D.N.; Ahmad, T.; Narsaiah, K. Recent Trends in the Use of Natural Antioxidants for Meat and Meat Products. Compr. Rev. Food Sci. Food Saf. 2015, 14, 796-812. [CrossRef]

14. Duthie, G.; Campbell, F.; Bestwick, C.; Stephen, S.; Russell, W. Antioxidant effectiveness of vegetable powders on the lipid and protein oxidative stability of cooked Turkey meat patties: Implications for health. Nutrients 2013, 5, 1241-1252. [CrossRef] [PubMed]

15. Rey, A.I.; Hopia, A.; Kivikari, R.; Kahkonen, M. Use of natural food/plant extracts: Cloudberry (Rubus Chamaemorus), beetroot (Beta Vulgaris “Vulgaris") or willow herb (Epilobium angustifolium) to reduce lipid oxidation of cooked pork patties. LWT Food Sci. Technol. 2005, 38, 363-370. [CrossRef]

16. Račkauskiene, I.; Pukalskas, A.; Venskutonis, P.R.; Fiore, A.; Troise, A.D.; Fogliano, V. Effects of beetroot (Beta vulgaris) preparations on the Maillard reaction products in milk and meat-protein model systems. Food Res. Int. 2015, 70, 31-39. [CrossRef]

17. Montowska, M.; Fornal, E. Detection of peptide markers of soy, milk and egg white allergenic proteins in poultry products by LC-Q-TOF-MS/MS. LWT Food Sci. Technol. 2018, 87, 310-317. [CrossRef]

18. Heick, J.; Fischer, M.; Pöpping, B. First screening method for the simultaneous detection of seven allergens by liquid chromatography mass spectrometry. J. Chromatogr. A 2011, 1218, 938-943. [CrossRef]

19. Hoffmann, B.; Münch, S.; Schwägele, F.; Neusüß, C.; Jira, W. A sensitive HPLC-MS/MS screening method for the simultaneous detection of lupine, pea, and soy proteins in meat products. Food Control 2017, 71, $200-209$. [CrossRef] 
20. Sanchez-Monge, R.; Lopez-Torrejón, G.; Pascual, C.Y.; Varela, J.; Martin-Esteban, M.; Salcedo, G. Vicilin and convicilin are potential major allergens from pea. Clin. Exp. Allergy 2004, 34, 1747-1753. [CrossRef]

21. Gomaa, A.; Boye, J. Impact of irradiation and thermal processing on the immunochemical detection of milk and egg allergens in foods. Food Res. Int. 2015, 74, 275-283. [CrossRef]

22. Packer, V.G.; Melo, P.S.; Bergamaschi, K.B.; Selani, M.M.; Villanueva, N.D.M.; de Alencar, S.M.; Contreras-Castillo, C.J. Chemical characterization, antioxidant activity and application of beetroot and guava residue extracts on the preservation of cooked chicken meat. J. Food Sci. Technol. 2015, 52, 7409-7416. [CrossRef]

23. Monaci, L.; Pilolli, R.; De Angelis, E.; Godula, M.; Visconti, A. Multi-allergen detection in food by micro high-performance liquid chromatography coupled to a dual cell linear ion trap mass spectrometry. J. Chromatogr. A 2014, 1358, 136-144. [CrossRef] [PubMed]

24. Montowska, M.; Fornal, E. Absolute quantification of targeted meat and allergenic protein additive peptide markers in meat products. Food Chem. 2019, 274, 857-864. [CrossRef] [PubMed]

25. Ji, J.; Zhu, P.; Pi, F.; Sun, C.; Sun, J.; Jia, M.; Ying, C.; Zhang, Y.; Sun, X. Development of a liquid chromatography-tandem mass spectrometry method for simultaneous detection of the main milk allergens. Food Control 2017, 74, 79-88. [CrossRef]

26. Chen, Q.; Ke, X.; Zhang, J.S.; Lai, S.Y.; Fang, F.; Mo, W.M.; Ren, Y.P. Proteomics method to quantify the percentage of cow, goat, and sheep milks in raw materials for dairy products. J. Dairy Sci. 2016, 99, 9483-9492. [CrossRef] [PubMed]

27. Mallick, P.; Kuster, B. Proteomics: A pragmatic perspective. Nat. Biotechnol. 2010, 28, 695-709. [CrossRef] [PubMed]

28. Wu, G.; Clerens, S.; Farouk, M.M. LC MS/MS identification of large structural proteins from bull muscle and their degradation products during post mortem storage. Food Chem. 2014, 150, 137-144. [CrossRef]

29. Altschul, S.F.; Madden, T.L.; Schaffer, A.A.; Zhang, J.; Zhang, Z.; Miller, W.; Lipman, D.J. Swiss-Prot Protein Knowledgebase, release 47.3 Gapped BLAST and PSI-BLAST: A new generation of protein database search programs. Nucleic Acids Res 1997, 25, 3389-3402. [CrossRef]

Sample Availability: Samples of the compounds are not available from the authors.

(c) 2018 by the authors. Licensee MDPI, Basel, Switzerland. This article is an open access article distributed under the terms and conditions of the Creative Commons Attribution (CC BY) license (http:/ / creativecommons.org/licenses/by/4.0/). 